\title{
Dynamics of charged bulk viscous collapsing cylindrical source with heat flux
}

\author{
S. M. Shah ${ }^{\mathrm{a}}$, G. Abbas ${ }^{\mathrm{b}}$ \\ Department of Mathematics, The Islamia University of Bahawalpur, Bahawalpur 63100, Pakistan
}

Received: 10 February 2017 / Accepted: 6 April 2017 / Published online: 19 April 2017

(C) The Author(s) 2017. This article is an open access publication

\begin{abstract}
In this paper, we have explored the effects of dissipation on the dynamics of charged bulk viscous collapsing cylindrical source which allows the out-flow of heat flux in the form of radiations. The Misner-Sharp formalism has been implemented to drive the dynamical equation in terms of proper time and radial derivatives. We have investigated the effects of charge and bulk viscosity on the dynamics of collapsing cylinder. To determine the effects of radial heat flux, we have formulated the heat transport equations in the context of Müller-Israel-Stewart theory by assuming that thermodynamics viscous/heat coupling coefficients can be neglected within some approximations. In our discussion, we have introduced the viscosity by the standard (non-causal) thermodynamics approach. The dynamical equations have been coupled with the heat transport equation; the consequences of the resulting coupled heat equation have been analyzed in detail.
\end{abstract}

\section{Introduction}

The stars composed of some nuclear matter which is continuously gravitating and is attracted toward its center due to the gravitational interaction of its particles. This phenomenon in the theory of general reactivity is known as gravitational collapse. The description of this phenomenon is the main objective of the relativistic theories of gravity (including general relativity) [1-3]. Oppenheimer and Snyder [4] theoretically illustrated the process of collapse in 1939, they addressed the contraction of a highly idealized spherically symmetric dust cloud. They used the exterior and interior spacetimes of the Schwarzschild metric and a Friedman like solution, respectively. An enormous amount of contributions in the research of gravitational collapse have been added by Vaidya [5], who provided the exterior gravitational field of a stellar body send-

\footnotetext{
${ }^{a}$ e-mail: syedmunawarshah71@ @otmail.com

be-mail: ghulamabbas@iub.edu.pk
}

ing out radiations. Misner and Sharp [6,7] studied a perfect fluid spherically symmetric collapse and also some authors [8-20] considered it in different situations.

Rossland [21] proved that the atoms are converted into ions with great strength and the law of central force should be observed by the forces between the free particles. Its order of magnitude should be greater than that of remaining forces acting between neutral atoms. The effect of electrical forces is fairly large if the star is built of heavy elements with 1.5 times the solar mass and a mean molecular weight 2.8 unit. Eddington [22] explored the fact that, in the internal electrical field of star, the electric potential $\phi$ directly relates the gravitational potential $\psi$, the mass $m$ and charge $e$ of a proton, a scalar parameter $\alpha$ affected by the density $n_{i}$ of the ions, the atomic weight $A_{i}$ of the ions and the effective charge $e Z_{i}$. Mitra [23] introduced the fact that the formation and evolution of stars would happen due to gravitational collapse, which is a high energy dissipating process and can be characterized by two respective cases: the free streaming approximation and the diffusion approximation. In the free streaming approximation case, Tewari added some models [24-26] by the solution of the Einstein field equation with a different approach. A number of distinguished researchers such as Bonner et al. [27], Bowers and Liang [28], de Oliveria [29], Mahraj and Govender [30], Ivanov [31] and Phinheiro and Chan [32] discussed many realistic models in the diffusion approximation with anisotropy, inhomogeneity, viscosity, and an electromagnetic field, and one also addressed the different dissipative processes analytically.

Since then, a huge amount of literature [8-20] on gravitational collapse considered the spherical symmetry of the star, which is the simplest geometry. In order to determine a realistic model of gravitational collapse, it would be interesting to study the dynamics of a collapsing star with a nonspherical background. It would be implied by the existence of gravitational waves that cylindrical and plane symmetries are more important for a non-spherical background. The cylin- 
drical sources may serve as a test bed for numerical relativity, quantum gravity, and for probing the cosmic censorship and hoop conjecture, among other important issues, and they represent a natural tool to seek the physics that lies behind the two independent parameters in the Levi-Civita metric [34]. Herrera et al. [33] have discussed gravitational collapse and junction/interface criteria for a gravitating source which has cylindrical geometry. Sharif and Ahmad [35] have predicted that gravitational radiations can be emitted during a gravitational collapse of two perfect fluids. Di Prisco et al. [36] studied the shear-free conditions and cylindrical gravitational waves by taking the Einstein-Rosen spacetime in the exterior of a general non-static cylindrical spacetime. Nakao and Morisawa [37] have explored the gravitational radiations from the collapse of a hollow cylinder.

Since Einstein and Rosen [38] initially predicted cylindrical gravitational waves theoretically, the observational evidence of gravitational waves through advance detectors such as LIGO [39] and GEO [40] has motivated researchers to study the cylindrically symmetric gravitating source. The formation of a naked singularity during the generic gravitational collapse would be expected during a cylindrical gravitational collapse. Several numerical approximations [41] depict the emission of gravitational cylindrical waves from a cylindrical gravitating source. These results have been verified analytically by Nakao and Morisawa [42]. During the recent years, many attempts [43-45] have been made to study the dynamics of collapsing cylindrical sources, but all these involve cylindrical spacetimes which are very similar to spherical spacetimes. In the current study, we have taken the nontrivial cylindrical spacetime.

In the present study, we have considered the two types of dissipation processes, heat dissipation associated to the radial heat flux and bulk viscosity. These both dissipative terms have been included in the stress energy tensor of the gravitating source. In order to see the effects of these terms on the dynamics of the collapse, we used the heat transport equations in the context of Müller-Israel-Stewart theory [46-49]. Such equations provide the physically reasonable heat transportation process as compared to LandauEckart approach [50,51] (by neglecting the thermodynamics viscous/heat coupling coefficients). The bulk viscosity has been described according to standard (non-causal) irreversible thermodynamics approach in the stress energy tensor of the gravitating source. The inclusion of the bulk viscosity in the fluid implies that we are assuming the relativistic Stokes equations, which corresponds to the irreversible thermodynamics. This equation does not satisfy causality, because implicitly it is assumed that the corresponding bulk viscosity relaxation time vanishes, and this assumption is valid within some approximations.

The plan of the paper is as follows: in Sect. 2 we present a cylindrical source and the field equations. The dynamical equations with Misner-Sharp approach have been presented in Sect. 3. The derivation of heat transport equation and its coupling is given Sect. 4. The last section is devoted to a summary of the results of this paper.

\section{Gravitating source and field equations}

In this section, we shall briefly introduce matter source, geometry of star for both interior and exterior regions and the field equations for the charged radiating bulk viscous source. The cylindrically symmetric spacetime [52] is

$\mathrm{d} s_{-}^{2}=-X^{2}(r, t) \mathrm{d} t^{2}+Y^{2}(r, t) \mathrm{d} r^{2}+R^{2}(r, t) \mathrm{d} \theta^{2}+\mathrm{d} z^{2}$,

where $-\infty \leq t \leq \infty, 0 \leq r,-\infty \leq z \leq \infty, 0 \leq \theta \leq 2 \pi$.

Inside the cylindrical star, we take a charged, anisotropic, bulk viscous fluid with radial heat flux, which has the following form of energy momentum tensor:

$$
\begin{aligned}
T_{\alpha \beta}= & \left(\mu+P_{r}\right) V_{\alpha} V_{\beta}-\left(P_{r}-P_{z}\right) S_{\alpha} S_{\beta}+\left(P_{r}-P_{\theta}\right) \chi_{\alpha} \chi_{\beta} \\
& -\left(g_{\alpha \beta}+V_{\alpha} V_{\beta}\right) \xi \Theta+q_{\alpha} V_{\beta}+V_{\alpha} q_{\beta}+P_{r} g_{\alpha \beta} \\
& +\frac{1}{4 \pi}\left(F_{\alpha}^{\gamma} F_{\beta \gamma}-\frac{1}{4} F^{\gamma \delta} F_{\gamma \delta} g_{\alpha \beta}\right),
\end{aligned}
$$

where $\mu$ is the energy density, $P_{r}$ is the pressure perpendicular to $z$ direction, $P_{\theta}$ is the pressure in the $\theta$ direction, $P_{z}$ is the pressure in $z$ direction, $V_{\alpha}$ is the four-velocity, $\xi$ is the coefficient of the bulk viscosity, $\Theta$ is the expansion scalar and $q_{\alpha}$ is the radial heat flux. Also, $F_{\alpha \beta}=-\phi_{\alpha, \beta}+\phi_{\beta, \alpha}$ is the Maxwell field tensor with four-potential $\phi_{\alpha}$. Moreover, $S_{\alpha}$ and $\chi_{\alpha}$ are the unit four-vectors, which satisfy the following relations:

$$
\begin{aligned}
& \chi^{\alpha} \chi_{\alpha}=S^{\alpha} S_{\alpha}=1, \quad V^{\alpha} V_{\alpha}=-1, \\
& V^{\alpha} S_{\alpha}=S^{\alpha} \chi_{\alpha}=V^{\alpha} \chi_{\alpha}=0 .
\end{aligned}
$$

The four-vector velocity $V_{\alpha}$ and four-vectors $\chi_{\alpha}$ and $S_{\alpha}$ can be defined as follows:

$\chi_{\alpha}=R \delta_{\alpha}^{2}, \quad v_{\alpha}=-X \delta_{\alpha}^{0}, \quad S_{\alpha}=\delta_{\alpha}^{3}$.

The Maxwell field equations are

$F_{; \beta}^{\alpha \beta}=4 \pi J^{\alpha}, \quad F_{[\alpha \beta ; \gamma]}=0$,

where $J_{\alpha}$ is the four-current. It is assumed that there exists only a non-vanishing electric scalar potential and the magnetic vector potential will be zero; then the four-potential takes the following form:

$\phi_{\alpha}=\phi \delta_{\alpha}^{0}, \quad J^{\alpha}=\zeta V^{\alpha}$,

where $\zeta(r, t)$ is the charge density and $\phi(r, t)$ is the electric scalar potential. 
The expansion scalar is

$\Theta=\frac{1}{X}\left(\frac{2 \dot{Y}}{Y}+\frac{\dot{R}}{R}\right)$

where a dot and a prime denote differentiation with respect to $t$ and $r$, respectively.

The set of Einstein-Maxwell field equations is

$$
\begin{gathered}
\kappa\left(\mu-\frac{\pi}{2} E^{2}\right) X^{2}=\frac{\dot{Y} \dot{R}}{Y R}+\left(\frac{X}{Y}\right)^{2}\left(\frac{X^{\prime} R^{\prime}}{X R}-\frac{R^{\prime \prime}}{R}\right), \\
\kappa q X Y^{2}=\frac{\dot{R}^{\prime}}{R}-\frac{\dot{Y} R^{\prime}}{Y R}-\frac{\dot{R} X^{\prime}}{R X}, \\
\begin{aligned}
\kappa\left(P_{r}-\xi \Theta+\frac{\pi}{2} E^{2}\right) Y^{2}= & \frac{X^{\prime} R^{\prime}}{X R}+\left(\frac{Y}{X}\right)^{2}\left(-\frac{\ddot{R}}{R}+\frac{\dot{X} \dot{R}}{X R}\right), \\
\kappa\left(P_{\theta}-\xi \Theta-\frac{\pi}{2} E^{2}\right)= & \left(\frac{1}{X Y}\right)\left(\frac{\dot{X} \dot{Y}}{X^{2}}-\frac{X^{\prime} Y^{\prime}}{Y^{2}}-\frac{\ddot{Y}}{X}+\frac{X^{\prime \prime}}{Y}\right), \\
\kappa\left(P_{z}-\xi \Theta-\frac{\pi}{2} E^{2}\right)= & -\frac{\ddot{Y}}{X^{2} Y}+\frac{X^{\prime \prime}}{X Y^{2}}-\frac{\ddot{R}^{2}}{X^{2} R}-\frac{X^{\prime} Y^{\prime}}{X Y^{3}} \\
& +\frac{\dot{X}}{X^{3}}\left(\frac{\dot{R}}{R}+\frac{\dot{X}}{X}\right)-\frac{R^{\prime}}{Y^{2} R}\left(\frac{Y^{\prime}}{Y}+\frac{X^{\prime}}{X}\right) \\
& \quad-\frac{\dot{Y} \dot{R}}{X^{2} Y R}+\frac{R^{\prime \prime}}{Y^{2} R},
\end{aligned}
\end{gathered}
$$

where $E(r, t)=\frac{\hat{Q}(r)}{2 \pi R}$ with total charge $\hat{Q}(r)=4 \pi \int_{0}^{r} \zeta Y R \mathrm{~d} r$.

Analogous to the Misner-Sharp mass in spherically symmetry, Thorne [38] introduced the mass function for cylindrical spacetime in terms of the gravitational C-energy per unit length of the cylinder. The specific energy $m(r, t)$ in the presence of an electromagnetic field is [53]

$m(r, t)=\frac{l}{8}\left[1+\left(\frac{\dot{R}}{X}\right)^{2}-\left(\frac{R^{\prime}}{Y}\right)^{2}\right]+\frac{\hat{Q}^{2} l^{2}}{2 R}$.

Here $l$ is the constant specific length of the cylinder.

Let $\Sigma$ be a boundary surface, which separates the interior region (defined in Eq. (1)) from the exterior region, the exterior region is described for a cylindrically symmetric manifold in the retarded time coordinate by [52]

$$
\begin{aligned}
\mathrm{d} s_{+}^{2}= & -\left(-\frac{2 M(\nu)}{\tilde{R}}+\frac{\tilde{q}^{2}(\nu)}{\tilde{R}^{2}}\right) \mathrm{d} \nu^{2} \\
& -2 \mathrm{~d} \nu \mathrm{d} \tilde{R}+\tilde{R}^{2}\left(\mathrm{~d} \theta^{2}+\gamma^{2} \mathrm{~d} Z^{2}\right),
\end{aligned}
$$

where $M(v)$ and $\tilde{q}(v)$ are the mass and the charge, respectively, and $\gamma^{2}=-\frac{\Lambda}{3}, \Lambda$ is the cosmological constant. Using the continuity of line elements and extrinsic curvature of line elements given by Eqs. (1) and (11) and the field equations, we get [53]

$$
\begin{aligned}
P_{r}-\xi \Theta & ={ }^{\Sigma}(q Y), \quad m-M={ }^{\Sigma} \frac{l}{8}, \\
\hat{Q}^{2} l^{2} & ={ }^{\Sigma} \tilde{q}^{2}, \quad l={ }^{\Sigma} 4 \tilde{R} .
\end{aligned}
$$

These are the necessary conditions for the smooth matching of internal and external geometries of cylindrical stars over the hypersurface $\Sigma$. For the assumed cylindrical source the difference of $M$ and $m$ (specific energy) is non-zero in general and the constraint $l={ }^{\Sigma} 4 \tilde{R}$ must be satisfied over $\Sigma$.

\section{Dynamical equations}

According to Misner and Sharp [6,7], we introduce the proper time derivative $D_{t}$ as follows:

$D_{t}=\frac{1}{X} \frac{\partial}{\partial t}$

The velocity $U$ of the fluid collapse may be stated in terms of Eq. (13) as

$U=D_{t} R<0 \quad$ (in the case of collapse).

Hence Eq. (10) yields

$\frac{\dot{R}}{Y}=\left(1+U^{2}-\frac{8 m}{l}+\frac{4 \hat{Q}^{2} l}{R}\right)^{\frac{1}{2}}=\hat{E}$

where $\hat{E}$ is the energy of an element of the fluid that undergoes collapse. The proper time derivative of the mass function described in Eq. (10) takes the following form:

$D_{t} m=l\left(\frac{\dot{R} \ddot{R}}{4 X^{3}}-\frac{\dot{R}^{2} \dot{X}}{Y X^{4}}-\frac{R^{\prime} \dot{R}^{\prime}}{4 Y^{2} X}+\frac{R^{\prime 2} \dot{Y}}{4 X Y^{3}}\right)-\frac{\dot{R} \hat{Q}^{2} l^{2}}{2 X R^{2}}$.

Using Eqs. (5) and (7) and $E=\frac{\hat{Q}}{2 \pi R}$, we obtain

$D_{t} m=-2 \pi l\left(\hat{E} q B+U\left(P_{r}-\xi \Theta\right)\right) R$.

These equations provide the rate of change of the total energy available inside the cylinder of radius $R$. Here, we briefly explain the effect of each term on the change of total internal energy, on the right hand side of the above equation with the term $\hat{E} q B$ being the multiple of negative sign. This stands for the amount of heat energy leaving the surface of the cylindrical star. In other words, the out-flow of heat from the collapsing system reduces the total energy of the system. In the second term $U\left(P_{r}-\xi \Theta\right)<0$ (as $U<0$, and $\Theta<0$ due to collapse and $\xi>0$ ), hence this having a pre-factor $-2 \pi$, increases the energy inside the collapsing source. The proper radial derivative $D_{\mathrm{R}}$ is used to address the dynamics of the collapsing system, which is defined as follows:

$D_{\mathrm{R}}=\frac{1}{R^{\prime}} \frac{\partial}{\partial r}$. 
Using Eqs. (10) and (18), we have

$D_{\mathrm{R}} m=\frac{l}{R^{\prime}}\left[\frac{\dot{R} \dot{R}^{\prime}}{4 X^{2}}-\frac{\dot{R}^{2} X^{\prime}}{4 X^{3}}-\frac{R^{\prime} R^{\prime \prime}}{4 Y^{2}}+\frac{Y^{\prime} R^{\prime 2}}{4 Y^{3}}+\frac{l \hat{Q} \hat{Q}^{\prime}}{R}-\frac{l \hat{Q}^{2} R^{\prime}}{2 R^{2}}\right]$.

Now Eqs. (5), (6) and (19) yield

$D_{\mathrm{R}} m=2 \pi R l\left(4 \mu+\frac{U}{\hat{E}} q Y\right)+\frac{\hat{Q} \hat{Q}^{\prime} l^{2}}{R R^{\prime}}-\frac{\hat{Q}^{2} l^{2}}{R^{2}}$.

This expression yields the change in total energy contained inside the various cylindrical surfaces of different radii. The term $4 \mu+\frac{U}{\hat{E}} q Y$ increases the energy as for the physically realistic fluid $\mu>0$, although it is affected by the heat flux and $U<0$ reduces $\mu$. The second term implies the presence of an electromagnetic field inside the gravitating source. After the integration of Eq. (20), we obtain

$m=\int_{0}^{R} 2 \pi R l\left(4 \mu+\frac{U}{\hat{E}} q Y\right) \mathrm{d} R+\frac{\hat{Q}^{2} l^{2}}{2 R}-\frac{l^{2}}{2} \int_{0}^{R} \frac{\hat{Q}^{2}}{R^{2}} \mathrm{~d} R$.

Here, we have assumed that $m(0)=0$.

Now we obtain $D_{t} U$, which is the acceleration of the collapsing matter inside the $\Sigma$. From Eq. (13), we get the following relation:

$D_{t} U=\frac{1}{X} \frac{\partial}{\partial t}\left(\frac{\dot{R}}{X}\right) \Rightarrow D_{t} U=\frac{\ddot{R}}{X^{2}}-\frac{\dot{R} \dot{X}}{X^{3}}$.

The above equation with Eq. (7) gives

$$
\begin{aligned}
D_{t} U= & -\left(\frac{m}{R^{2}}+8 \pi\left(P_{r}-\xi \Theta\right) R\right)+\frac{X^{\prime} \hat{E}}{X Y}+\frac{\hat{Q}^{2}}{R}\left(\frac{l^{2}}{2 R^{2}}-1\right) \\
& +\frac{l}{8 R^{2}}\left(1+U^{2}-\hat{E}^{2}\right) .
\end{aligned}
$$

By the conservation law $\left(T_{; \beta}^{\alpha \beta}=0\right)$, we deduce the following dynamical equations:

$$
\begin{aligned}
& P_{r}^{\prime}+\frac{\dot{q} Y^{2}}{X}-\left(P_{\Theta}-P_{r}\right) \frac{R^{\prime}}{R}+\frac{q Y^{2}}{X}\left(\frac{\dot{R}}{R}+\frac{3 \dot{Y}}{Y}\right) \\
& +\left(P_{r}+\mu\right) \frac{X^{\prime}}{X}-\xi \Theta^{\prime}+\frac{X^{\prime}}{X} \xi \Theta \\
& +\left(\frac{\hat{Q}}{R^{2}}\right)\left(E^{\prime} R-R^{\prime} E\right)=0 .
\end{aligned}
$$

Using the value of $\frac{X^{\prime}}{X}$ from Eq. (24) in Eq. (23) and considering the field equations, after some algebra we obtain

$$
\begin{aligned}
\left(P_{r}\right. & +\mu-\xi \Theta) D_{t} U \\
= & -\left(\mu+P_{r}-\xi \Theta\right)\left[\frac{m}{R^{2}}+8 \pi R\left(P_{r}-\xi \Theta\right)+\frac{l^{2} \hat{Q}^{2}}{2 R^{3}} \frac{\hat{Q}^{2}}{R}+\frac{l U^{2}}{8 R^{2}}\right] \\
& -\hat{E}^{2}\left[\frac{P_{r}}{R}-\frac{P_{\theta}}{R}-\frac{\hat{Q}^{2}}{\pi R^{3}}+\frac{l\left(\mu+P_{r}-\xi \Theta\right)}{8 R^{2}}\right] \\
& -\hat{E}\left[\frac{P_{r}^{\prime}}{Y}+\frac{3 q \dot{Y}}{X}+Y D_{t} q+q Y \frac{\dot{R}}{X R}-\frac{\xi \Theta^{\prime}}{Y}+\frac{\hat{Q} \hat{Q}^{\prime}}{2 \pi Y R^{2}}\right] .
\end{aligned}
$$

The factor $\left(P_{r}+\mu-\xi \Theta\right)$ being a multiple of the acceleration $D_{T} U$ plays the role of an effective inertial mass density, while the same factor on the right hand side before the square bracket is the passive gravitational mass density. This factor is affected by the radial pressure and bulk viscosity, but it is independent of the electric charge. The first square bracket on the right hand side shows the effects of dissipation and charge on the dynamical process. The second square bracket gives the effects of the local anisotropy, electric charge and gravitational mass density. In the last square bracket $P_{r}^{\prime}$ is the pressure gradient and the terms involving $q$, $\xi$ and $\hat{Q}$ explain the collective effects of dissipation and the electromagnetic field on the hydrodynamics of the collapsing source. The consequences of $D_{t} q$ will be dealt with in the next section by deriving the heat transport equation and then performing the possible coupling of the dynamical equation with the resulting heat transport equation.

\section{Heat transport equation}

As already mentioned in the introduction, we shall use a transport equation that comes from the Müller-IsraelStewart [46-49] second order phenomenological theory for dissipative fluids (by neglecting the thermodynamics viscous/heat coupling coefficients). Since we have introduced the bulk viscosity in a fluid source, we have to take accordingly the full causal approach as discussed in [54-59], but, for the sake of simplicity, we neglect the thermodynamics viscous/heat coupling coefficients and only take into account the only transportation of heat flux governed bythe Cattaneo type equation [60] (leading to a hyperbolic equation for the propagation of a thermal perturbation). Thus according to $[12,14]$, the transport equation for the heat flux is

$$
\begin{aligned}
\tau h^{\alpha \beta} V^{\gamma} q_{\beta ; \gamma}+q^{\alpha}= & -K h^{\alpha \beta}\left(T_{, \beta}+T a_{\beta}\right) \\
& -\frac{1}{2} K T^{2}\left(\frac{\tau V^{\beta}}{K T^{2}}\right)_{; \beta} q^{\alpha} .
\end{aligned}
$$

In the above equation $h^{\alpha \beta}$ denotes the projection onto the space orthogonal to $V^{\beta}, K$ is the thermal conductivity and $T$ and $\tau$ are temperature and relaxation time, respectively. With the symmetry of the given interior spacetime, the heat transport equation has the following form as regards the independent component: 


$$
\begin{aligned}
Y D_{t} q= & -\frac{K Y T^{\prime}}{\tau}-\frac{K T Y}{\tau}\left(\frac{X^{\prime}}{X}\right)-\frac{1}{2 \tau} K T^{2} Y^{3}\left(\frac{\tau}{K T^{2}}\right) \dot{q} \\
& +\frac{\dot{Y} q}{X}-\frac{Y q}{X \tau}-\frac{3}{2 X} \dot{Y} q Y .
\end{aligned}
$$

Applying the value of $\frac{X^{\prime}}{X}$ from Eq. (23) in the above equation, we get

$$
\begin{aligned}
Y D_{t} q= & -\frac{K Y T^{\prime}}{\tau}-\frac{K T Y^{2}}{\tau \hat{E}} D_{t} U-\frac{K T Y^{2}}{\tau \hat{E}} \\
& \times\left(\frac{m}{R^{2}}+8 \pi R\left(P_{r}-\xi \theta\right)-\frac{\hat{Q}^{2} l^{2}}{2 R^{3}}+\frac{\hat{Q}^{2}}{R}+\frac{l\left(1+U^{2}\right)}{8 R^{2}}\right) \\
& -K T Y^{2}\left(\frac{l \hat{E}}{8 \tau R^{2}}\right)-\frac{1}{2 \tau} K T^{2} Y^{3}\left(\frac{\tau}{K T^{2}}\right) q+\frac{\dot{Y} q}{X} \\
& -\frac{Y q}{\tau X}-\frac{3}{2 X} \dot{Y} q Y .
\end{aligned}
$$

After substituting the value of $Y D_{t} q$ in Eq. (25), we have

$$
\begin{aligned}
( & \left.\left(P_{r}+\mu-\xi \Theta\right)-\frac{K T Y^{2}}{\tau}\right) D_{t} U \\
= & -\left(P_{r}+\mu-\xi \Theta\right)\left(\frac{m}{R^{2}} 8 \pi R\left(P_{r}-\xi \Theta\right)-\frac{\hat{Q}^{2} l^{2}}{2 R^{3}}\right. \\
& \left.+\frac{\hat{Q}^{2}}{R}+\frac{l\left(1+U^{2}\right)}{8 R^{2}}\right)\left(1-\frac{K T Y^{2}}{\tau\left(P_{r}+\mu-\xi \Theta\right)}\right) \\
& -\hat{E}^{2}\left[\frac{P_{r}-P_{\theta}}{R}-\frac{\hat{Q}^{2}}{\pi R^{3}}+\frac{l\left(P_{r}+\mu-\xi \Theta\right)}{8 R^{2}}+\frac{l K T Y^{2}}{8 R^{2} \tau}\right] \\
& -\hat{E}\left[\frac{P_{r}^{\prime}}{Y}+\frac{4 q \dot{Y}}{X}-\frac{K Y T^{\prime}}{\tau} \frac{1}{2 \tau} K T^{2} Y^{3}\left(\frac{\tau}{K T^{2}}\right) q\right. \\
& \left.-\frac{q Y}{X \tau}-\frac{3 q \dot{Y} Y}{2 X}\right] .
\end{aligned}
$$

Equation (29) may be written as

$$
\begin{aligned}
& \left(P_{r}+\mu-\xi \Theta\right)(1-\alpha) D_{t} U \\
& =F_{\text {grav }}(1-\alpha)+F_{\text {hyd }}-\hat{E}\left[\frac{P_{r}^{\prime}}{Y}+\frac{4 q \dot{Y}}{X}-\frac{K Y T^{\prime}}{\tau}\right. \\
& \left.\quad-\frac{1}{2 \tau} K T^{2} Y^{3}\left(\frac{\tau}{K T^{2}}\right) q-\frac{q Y}{X \tau}-\frac{3 q \dot{Y} Y}{2 X}\right] .
\end{aligned}
$$

Here, $F_{\text {grav }}, F_{\text {hyd }}$ and $\alpha$ are defined by

$$
\begin{aligned}
F_{\text {grav }}= & -\left(P_{r}+\mu-\xi \Theta\right)\left(\frac{m}{R^{2}}+8 \pi R\left(P_{r}-\xi \Theta\right)-\frac{\hat{Q}^{2} l^{2}}{2 R^{3}}\right. \\
& \left.+\frac{\hat{Q}^{2}}{R}+\frac{l\left(1+U^{2}\right)}{8 R^{2}}\right), \\
F_{\text {hyd }}= & -\hat{E}^{2}\left[\frac{P_{r}}{R}-\frac{P_{\theta}}{R}-\frac{\hat{Q}^{2}}{\pi R^{3}}+\frac{l\left(\mu+P_{r}-\xi \Theta\right)}{8 R^{2}}\right], \\
\alpha= & \frac{K T Y^{2}}{\tau\left(P_{r}+\mu-\xi \Theta\right)} .
\end{aligned}
$$

From Eq. (30), it is noted how dissipation affects the final stage of the charged collapsing cylinder. This fact was investigated for the first time in [61], when the authors discussed the thermal conduction in systems out of hydrostatic equilibrium. They analyzed the fact that the evolution of the gravitating source depends on the parameter $\alpha$ (which is defined in terms of the thermodynamic variables), further for the validity of causality, the constraints on $\alpha$ have been determined in that work.

It is clear that the left hand side of Eq. (30) will be zero as $\alpha \rightarrow 1$, which confirms that the effective inertial mass density of the fluid element tends to zero. Further, we observe that the inertial mass will be decreased as $\alpha$ exceeds than 1 . Moreover, $F_{\text {grav }}$, being a multiple of $(1-\alpha)$, is affected by this factor. Also, it is evident that both inertial mass and gravitational attraction are affected by the same factor $(1-\alpha)$. In other words, we can say that this equation satisfies the equivalence principle and we would like to point out that the factor $(1-\alpha)$ has no effects on $F_{\text {hyd }}$. One may observe that a collapsing cylinder would evolve in such a way that the value of $\alpha$ keeps on increasing and attains a critical value of 1 . With the passage of time during collapse the rapid decrease in the force of gravity may gradually result in altering the physical effects of the right hand side of Eq. (30). As is clear from the definition of $\alpha$, it is inversely related to the effective inertial mass density, so as long as $\alpha$ increases from 1, then there would be a decrease in the inertial mass density. Physically, it is only possible when the gravitating source depicts the bouncing behavior. The factor $(1-\alpha)$ does not depend on the charge parameter but it heavily depends on the bulk viscosity, which is explicitly clear from Eq. (32). Further, one can see the dependence of the factor $(1-\alpha)$ on the dissipative variables when a full causal approach [62] is used to discuss the dynamics of dissipative collapse (see Eq. (54) of [62]).

\section{Conclusion}

The cylindrically symmetric systems which combine translations along the axis are exactly known to general relativists. The study of such systems was started by Weyl [63] and Levi-Civita [34] in the early 20th century immediately after the birth of the Einstein theory of relativity. In the beginning physicists were interested in finding the gravitating objects that are exactly axially symmetric. The realistic fluids are very important in the modeling of astronomical objects. So, one cannot ignore the effects of dissipation during the gravitational collapse.

Here, we discuss the gravitational collapse of charged radiating cylindrically symmetric stars. To this end, we formulated the Einstein field equations and conservation equation for a non-static charged bulk viscous heat conducting anisotropic cylindrically symmetric source. Using the Misner 
and Sharp formalism, the dynamical equations are derived. Further, we have considered the two types of dissipation processes, heat dissipation associated to the radial heat flux and bulk viscosity. In order to see the effects of these terms on the dynamics of the collapse, we have excluded thermodynamics viscous/heat coupling coefficients in the heat transport equations in the context of Müller-Israel-Stewart theory [46-49].

The inclusion of the bulk viscosity in the fluid implies that we are assuming the relativistic Stokes equations, which corresponds to irreversible thermodynamics. This equation does not satisfy causality, because implicitly it is assumed that the corresponding bulk viscosity relaxation times vanishes. But this assumption is sensible, because within some approximations, such relation times could be neglected. A full causal approach to the dynamics of dissipative collapse has been analyzed with significant consequences in [62], without excluding the thermodynamics viscous/heat coupling coefficients for the heat flux and bulk viscosity. As an implication of their analysis to astrophysical scenario, they pointed out that in a pre-supernova event, the dissipative parameters (particularly the thermal conductivity) would be so large as to produce a significant decreasing in the force of gravity which leads to the reversal of the collapse. We would like to mention that we have introduced the bulk viscosity by the standard (non-causal) irreversible thermodynamics approach, so we have used the partially causal approach (the thermodynamics viscous/heat coupling coefficients have been excluded) to discuss the dynamics of the dissipative source considered. Further, the form of Eqs. (17), (23), (24), (25), (29), (30), and (32) depends on the standard (non-causal) irreversible thermodynamics approach, which we have used in the present analysis. If one considers the full causal approach to the discussion of the dynamics of dissipative gravitational collapse as in [62], then in the present case the term $-\xi \Theta$ in Eqs. (17), (23), (24), (25), (29), (30), and (32) will be replaced by a dissipative variable $\Pi$.

Finally, in our analysis, it has been investigated that during the evolution of the cylindrical star, charge, bulk viscosity and anisotropic stresses reduce the energy of the system and we conclude the following.

- The out flow of heat from the collapsing cylindrical star reduces the total energy of the system.

- The bulk viscosity reduces the radial pressure of the collapsing fluid.

- The bulk viscosity and charge of the fluid would affect the rate of collapse prominently.

- Active/passive gravitational mass density is affected by the bulk viscosity and it is independent of the electromagnetic field.

- In Eq. (30), the factor $(1-\alpha)$ would explain the possible evolutionary stages of the charged dissipative cylinder.
- The term $\alpha$ is inversely related to the gravitational mass density, which is affected by the bulk viscosity, while it is linearly related to temperature of the fluid.

- The inclusion of the bulk viscosity would increase the value of $\alpha$.

- For $\alpha<1, \alpha>1$ and $\alpha=1$, we have an expanding, collapsing and bouncing behavior of the fluid distribution, respectively.

Acknowledgements The constructive comments and suggestions of an anonymous referee are highly appreciated.

Open Access This article is distributed under the terms of the Creative Commons Attribution 4.0 International License (http://creativecomm ons.org/licenses/by/4.0/), which permits unrestricted use, distribution, and reproduction in any medium, provided you give appropriate credit to the original author(s) and the source, provide a link to the Creative Commons license, and indicate if changes were made.

Funded by $\mathrm{SCOAP}^{3}$.

\section{References}

1. E.A. Milne, MNRAS 91, 4 (1930)

2. S. Chandrasekhar, MNRAS 91, 456 (1931)

3. S. Chandrasekhar, MNRAS 95, 207 (1935)

4. J.R. Oppenheimer, H. Snyder, Phys. Rev. 56, 455 (1939)

5. P.C. Vaidya, Proc. Indian Acad. Sci. A33, 264 (1951)

6. C.W. Misner, D. Sharp, Phys. Rev. 136B, 571 (1964)

7. C.W. Misner, D. Sharp, Phys. Rev. 137B, 1360 (1965)

8. K. Lake, C. Hellaby, Phys. Rev. D 24, 3019 (1981)

9. N.O. Santos, MNRAS 216, 403 (1985)

10. L. Herrera, N.O. Santos, Phys. Rep. 286, 53 (1997)

11. L.Di Herrera, A. Prisco, J.R. Hernandez, N.O. Santos, Phys. Lett. A 237, 113 (1998)

12. L. Herrera, N.O. Santos, Phys. Rev. D 70, 084004 (2004)

13. L. Herrera, A. Di Prisco, J. Ospino, Gen Relativ. Gravity 44, 2645 (2012)

14. L. Herrera, Int. J. Modern Phys. D 15, 2197 (2006)

15. L. Herrera, A. Di Prisco, W. Barreto, Phys. Rev. D 73, 024008 (2006)

16. L. Herrera, N.O. Santos, A. Wang, Phys. Rev. D 78, 084026 (2008)

17. L. Herrera, A. Di Prisco, J. Martin, J. Ospino, N.O. Santos, O. Troconis, Phys. Rev. D 69, 084026 (2004)

18. R. Chan, MNRAS 316, 588 (2000)

19. W.B. Bonor, A.R.G. de Oliveira, N.O. Santos, Phys. Rep. 181, 269 (1989)

20. A. Di Prisco, L. Herrera, G. Le Denmat, A.H. Maccullum, N.O. Santos, Phys. Rev. D 76, 064017 (2007)

21. S. Rosseland, MNRAS 84, 720 (1924)

22. A.S. Enddington, Internal Constitution of the Stars (Cambridge University Press, Cambridge, 1926)

23. A. Mitra, Phys. Rev. D 74, 024010 (2006)

24. B.C. Tewari, Astrophys. Space Sci. 149, 233 (1988)

25. B.C. Tewari, Indian J. Pure Appl. Math. 32, 504 (1994)

26. B.C. Tewari, Astrophys. Space Sci. 306, 273 (2006)

27. W.B. Bonnor, A.R.G. de Oliveira, N.O. Santos, Phys. Rev. Lett. 181, 269 (1989)

28. R.L. Bowers, E.P.T. Liang, Astrophys. J. 188, 657 (1974)

29. A.K.G. de Oleveiria, N.O. Santos, C.A. Kolassis, MNRAS 216 , $1001(1985)$

30. S.D. Maharaj, M. Govender, Int. J. Modern Phys. D 14, 667 (2005)

31. B.V. Ivanov, Int. J. Modern Phys. D 20, 319 (2011) 
32. G. Pinheiro, R. Chan, Gen. Relativ. Gravity 45, 243 (2013)

33. L. Herrera, M.A.H. MacCallum, N.O. Santos, Class. Quant. Gravity 24, 1033 (2007)

34. T. Levi-Civita, Atti Acc. Lincei Rend. 28, 101 (1919)

35. M. Sharif, Z. Ahmad, Gen. Relativ. Gravity 39, 1331 (2007)

36. A. Di Prisco, L. Herrera, G. Denmat, M.A.H. MacCallum, N.O. Santos, Phys. Rev. D 80, 064031 (2009)

37. K. Nakao, Y. Morisawa, Class. Quant. Gravity 21, 2101 (2004)

38. K.S. Thorne, Phys. Rev. 138, B251 (1965)

39. A. Abramovici et al., Science 256, 325 (1992)

40. H. Lück; the GEO600 Team: Class. Quantum Gravity 14, 1471 (1997)

41. T. Piran, Phys. Rev. Lett. 41, 1085 (1978)

42. K. Nakao, Y. Morisawa, Phys. Rev. D 71, 124007 (2005)

43. M. Sharif, S. Fatima, Gen. Relativ. Gravity 43, 127 (2011)

44. M. Sharif, G. Abbas, Astrophys. Space Sci. 335, 515 (2011)

45. S. Guha, R. Banerji, Int. J. Theor. Phys. 53, 2332 (2014)

46. I. Müller, Z. Phys. 198, 329 (1967)

47. W. Israel, Ann. Phys. NY 100, 310 (1976)

48. W. Israel, J. Stewart, Phys. Lett. A 58, 213 (1976)
49. W. Israel, J. Stewart, Ann. Phys. N Y 118, 341 (1979)

50. C. Eckart, Phys. Rev. 58, 919 (1940)

51. L. Landau, E. Lifshitz, Fluid Mechanics (Pergamon Press, London, 1959)

52. M. Sharif, M. Azam, JCAP 02, 043 (2012)

53. M. Sharif, M.Z. Bhatti, JCAP 10, 056 (2013)

54. D. Joseph, L. Preziosi, Rev. Modern Phys. 61, 41 (1989)

55. J. Casas-Vázquez, G. Lebon, Rep. Prog. Phys. 51, 1105 (1988)

56. Maartens, R.: arXiv:astro-ph/9609119

57. L. Herrera, D. Pavón, Phys. A 307, 121 (2002)

58. W. Hiscock, L. Lindblom, Ann. Phys. 151, 466 (1983)

59. D. Pavón, D. Jou, J. Casas-Vázquez, Ann. Inst. H Poincaré A 36, 79 (1982)

60. C. Cattaneo, Atti Semin. Mat. Fis. Univ. Modena 3, 3 (1948)

61. L. Herrera, A. Di Prisco, J. Hernández-Pastora, J. Martín, J. Martínez, Class. Quant. Gravity 14, 2239 (1997)

62. L. Herrera, A. Di Prisco, E. Fuenmayor, O. Troconis, Int. J. Modern Phys. D 18, 129 (2009)

63. H. Weyl, Ann. Phys. Lpz. 54, 117 (1917) 